\title{
I humanize, therefore I understand? Effects of explanations and humanization of intelligent systems on perceived and objective user understanding
}

\author{
Thao $\mathrm{Ngo}^{\mathrm{a}}$ and Nicole Krämer ${ }^{\mathrm{b}}$ \\ aResearch Training Group "User Centred Social Media", University of Duisburg-Essen, \\ Duisburg, Germany \\ ${ }^{\mathrm{b}}$ Social Psychology: Media and Communication, University of Duisburg-Essen, \\ Duisburg, Germany
}

Authors' Original Version (Preprint)

\begin{abstract}
Author Note
Thao Ngo: https://orcid.org/0000-0001-5147-8272
\end{abstract}

Nicole Krämer: https://orcid.org/0000-0001-7535-870X

Correspondence concerning this article should be addressed to Thao Ngo:

thao.ngo@uni-due.de 


\begin{abstract}
The functioning of intelligent systems can be opaque to users. Yet, users need to make informed choices about them. This work compares two knowledge mechanisms, i.e., ways for users to achieve an understanding of intelligent systems: explanation and humanization. In online experiment $(N=416)$, we compared the effects of a control condition without any explanation against a) a neutral and b) a humanized how-explanations as well as c) active humanization on (perceived and objective) user understanding and systems perceptions (trust, transparency, satisfaction, perceived usefulness). Our main finding: Explanations increased transparency and perceived understanding but not objective understanding. Active humanization, surprisingly, decreased objective understanding compared to the control condition suggesting inhibition of knowledge retrieval. Humanized how-explanation increased the perceived usefulness. We found no effects for trust and satisfaction. We conclude that explanations lead to a deceptive feeling of understanding. Explanations should consider the prior understanding to affect objective understanding.
\end{abstract}

Keywords: user understanding; explanation; humanization; intelligent system; algorithmic ranking; transparency 


\section{HUMANIZE, THEREFORE I UNDERSTAND?}

\section{Introduction}

Intelligent systems are ubiquitous in their application. For instance, as ranking algorithms, they determine which information users are exposed to on their search results or social media and news feed. As such, they can serve as gatekeepers to information (Cotter \& Reisdorf, 2020; Gillespie, 2014). Despite their tremendous importance, prior research highlights that users have sparse knowledge (Araujo et al., 2020) or idiosyncratic ideas about these systems' inner workings (DeVito et al., 2018; Eslami et al., 2016). Users might also have misconceptions about the possible inferences that intelligent systems can make (Hautea et al., 2020). This flawed understanding threatens the user's autonomy to make informed decisions about the use and interaction with intelligent systems. As a consequence, users can lack the possibility to scrutinize the system's outputs and overly rely on their decisions (Cotter \& Reisdorf, 2020; Pan et al., 2007). Therefore, the user understanding, i.e., what users know about the processing of (personal user) data, the algorithmic model, and the generation of specific outputs, is crucial to retain the users' autonomy (Cotter \& Reisdorf, 2020). Additionally, from a system developer's perspective, user understanding is a prerequisite for acceptance and interaction with intelligent systems (Norman, 1983; Yeomans et al., 2019). Prior research also highlights that users are interested in learning more about the inner workings of intelligent systems (Eslami et al., 2016; Lim \& Dey, 2009).

Therefore, this work concerns the question of how users can gain an understanding of intelligent systems. We compare two knowledge mechanisms which we define as ways through which users can achieve understanding about a particular intelligent system.

The first mechanism concerns explanations of intelligent systems. There are several types of textual and visual explanations that aim to increase algorithmic 


\section{HUMANIZE, THEREFORE I UNDERSTAND?}

transparency. They can affect the user understanding and perception of an intelligent system differently (Mohseni et al., 2020). In this work, we are specifically interested in the effects of how-explanations. These explanations describe intelligent algorithms' inner working and can increase user awareness and user understanding (Rader et al., 2018). We present neutral how-explanations and humanized how-explanations. The latter entails anthropomorphic descriptions of the system.

The second mechanism concerns a phenomenon that can occur when individuals face technology: Humanization (also called anthropomorphism) is the process of attributing human characteristics and states to non-human agents by using one's very own as an anchor (Epley et al., 2007). Humanization can be an inherent part of user understanding (Kodama et al., 2017; Ngo et al., 2020; Wu et al., 2019). Individuals tend to humanize, especially when systems appear unpredictable and they feel uncertain about their behavior (Epley et al., 2007; Waytz et al., 2014). Therefore, we suggest that humanization might occur particularly in the absence of explanations.

In this regard, we ask whether humanization can provide a deceptive feeling of user understanding. If so, we argue that humanization can be disadvantageous when users intend to gain an understanding of intelligent systems. Users might merely have the perception that they hold a correct understanding, while they actually do not.

Therefore, this study pursues two research goals: (1) We compare the effects of these two knowledge mechanisms (neutral how-explanation and humanization) and their combination (humanized how-explanations) on the perceived and objective user understanding. The distinction between perceived, i.e., the extent to which users feel that they have understood a system, and objective understanding are important. Prior research could already show that users may have an illusion of understanding when informing themselves using the Internet (Fisher et al., 2015). In this regard, our main 


\section{HUMANIZE, THEREFORE I UNDERSTAND?}

hypotheses are that explanations lead to a higher perceived and objective understanding. In comparison, humanization should only lead to a higher perceived understanding. Additionally, we (2) explore the effects of explanations and humanization on different system perceptions, including trust, transparency, satisfaction, and perceived usefulness.

To address these goals, we conducted a pre-registered online experiment choosing the news ranking algorithm of Google News as an example for intelligent systems. In particular, our study sought to answer the following questions:

- RQ1: How do explanations and humanization contribute to the perceived and objective user understanding of intelligent systems (in this case, Google News)?

- RQ2: How do explanations and humanization contribute to the perception of intelligent systems (trust, transparency, satisfaction, perceived usefulness)?

This work examines the effects of how-explanations and humanization on user understanding and highlights the danger of the discrepancy between perceived and objective user understanding. By understanding these effects, future research on algorithmic transparency can evaluate and improve explanations to ensure their effectiveness for user understanding.

\section{Background}

In the following sections, we first provide an overview of explanations and the underlying psychological mechanisms of humanization. We then derive our hypotheses based on the existing literature.

\section{Categories and effects of explanations}

Explanations serve to expand the user understanding of a particular subject (Keil, 2006). Researchers have distinguished explanations based on their content, 


\section{HUMANIZE, THEREFORE I UNDERSTAND?}

presentation format (textual or visual), and the provision mechanism (Gregor \& Benbasat, 1999). Concerning the content of explanations, they can be categorized as global (or model-centric) explanations, which provide general information about an algorithmic system, or local (or subject-centric) explanations, which are case-specific and based on the input data that is given to an algorithmic system (Darlington, 2013; Došilović et al., 2018; Edwards \& Veale, 2017). Another distinction can be made between how-explanations (white-box explanations) and why-explanations (black-box explanations; Friedrich and Zanker, 2011). How-explanations describe how an algorithmic system derives a particular outcome based on a specific input. Their purpose is to provide knowledge about how a particular task is done. Why-explanations, in turn, justify the reasoning behind a specific output (Darlington, 2013).

Still, it remains open how much and which kind of content should be included in an explanation. On the one hand, Nourani et al. (2019) showed that explanations need to be meaningful to be effective. On the other side, Eiband et al. (2019) showed that explanations that do not convey any new information can still increase trust similarly to meaningful explanations.

While one relevant goal of explanations is to provide transparency (Lipton, 2017), they can affect many other variables. Providing explanations increases user understanding of intelligent systems and can increase satisfaction and perceived usefulness (Dominguez et al., 2019; Lim et al., 2009). Explanations can also affect fairness perceptions of intelligent systems. For example, Dodge et al. (2019) found that local explanations reveal more fairness discrepancies in judging (fictive) defendant's reoffense risks than global explanations. Why-explanations have been found to increase benevolence perception and trust in recommender systems (Wang \& Benbasat, 2007; Zanker \& Ninaus, 2010). 


\section{HUMANIZE, THEREFORE I UNDERSTAND?}

In this study, we focus on how-explanations can increase benevolence and competence perception of recommender systems (Wang \& Benbasat, 2007) as well as the awareness of an intelligent algorithm's existence, the understanding of its performance, and its accountability (Rader et al., 2018). We deem this kind of explanation as particularly interesting for the user understanding as they provide information about the inner working of intelligent systems. As such, we assume that they potentially hold information that is unfamiliar to users. Therefore, they might increase the objective understanding more than other types of explanations.

\section{Consequences of humanization}

Individuals have the capability and tendency to humanize non-human agents (Epley et al., 2007). This has been consistently observed for all kinds of technology, including AI-enabled technology and robots (Blut et al., 2021; M. Li \& Suh, 2021). One central theory that explains and predicts the circumstances under which humanization occurs is the three-factor theory of humanization (Epley et al., 2007).

According to this theory, humanization is driven by three psychological key determinants: (1) the elicitation of agent knowledge, (2) effectance motivation, and (3) sociality motivation. Concerning the first determinant, Epley and colleagues (2007) proposed that humanization is an inductive inference process. In other words, individuals make conclusions about a non-human agent based on readily accessible knowledge. This knowledge can stem from two sources. Firstly, individuals apply knowledge about themselves or humans in general to the non-human agent. According to Epley and colleagues (2007), this first source is more likely to serve as a primary basis because it is richer and acquired earlier. Thus, it is more likely to be available when facing a non-human agent. Secondly, the knowledge can stem from the non- 


\section{HUMANIZE, THEREFORE I UNDERSTAND?}

human agent itself. For example, in human-robot interaction, inferences about a robot can be provoked through visual anthropomorphic features, such as the surface look (gender, skin, and eyelashes), body or facial features (Phillips et al., 2018). The humanization can also be triggered by verbal anthropomorphic features like voice and human-like language (M. Li \& Suh, 2021).

As the second determinant, the effectance motivation describes the "need to interact effectively with one's environment" (Epley et al., 2007, p. 866). Individuals wish to explain and predict the non-human agent's behavior, which, in turn, can reduce the feeling of uncertainty about the non-human agent. The third determinant, the sociality motivation, describes the need for social connections. Humanization satisfies this need by enabling individuals to build connections to non-human agents. Empirical studies confirm the predictions of the three-factor theory regarding both motivations. For example, the desire for control and chronic loneliness can affect the degree of humanization of animals, technology, and religious agents (Epley, Akalis, et al., 2008; Epley, Waytz, et al., 2008). Furthermore, the unpredictability of robots can increase humanization (Eyssel et al., 2011; Waytz et al., 2010).

Humanization can affect the user perception of non-human agents, attitudes, and behavior towards them robots (Blut et al., 2021; M. Li \& Suh, 2021). Human-like robots and speech agents seem to be more likable and associated with warmth and pleasure. They are also seen as more intelligent (Haas \& Moussawi, 2020). Moreover, humanization can increase technology acceptance, including the acceptance of augmented reality applications (Dominguez et al., 2019) and robots, when users have prior experience with similar technology (Goudey \& Bonnin, 2016).

Within the scope of this work, we aim to explore the effects of humanization on system perceptions. Regarding trust, so far, research has shown mixed results. Some 


\section{HUMANIZE, THEREFORE I UNDERSTAND?}

studies suggest no or a weak relationship between humanization and trust. For instance, a meta-analysis by Hancock et al. (2011) revealed a small effect of humanization on trust in robots. Instead, they found that the performance of a robot contributed more to trust perception. More recent studies showed that humanization could increase trust in human-like robots (de Visser et al., 2016; Natarajan \& Gombolay, 2020; van Pinxteren et al., 2019), autonomous vehicles (Forster et al., 2017; Waytz et al., 2014), and anthropomorphic presentations of brands (Aggarwal \& McGill, 2012; Golossenko et al., 2020).

Besides trust, we aim to investigate the effects on other user perceptions, in particular, transparency, satisfaction, and perceived usefulness. They are also defined as user beliefs that are influenced by the perceived system qualities (e.g., interaction adequacy or interface adequacy of a system; Pu et al., 2011). Yet, studies of humanization effects on user perceptions of intelligent algorithms are scarce to this date.

\section{Derivation of hypotheses}

We aim to compare the effects of how-explanations and humanizations on perceived understanding and objective user understanding. Additionally, we are curious how the combination of both knowledge mechanisms, thus humanized explanations, affects user understanding and system perceptions. We argue that humanized explanations can be understood as a more passive form of humanization, as the explanation itself entails anthropomorphic cues of a system. Therefore, individuals do not solely rely on their humanization capabilities.

How-explanations entail the steps which the algorithmic system takes to arrive at a specific output. Rader et al. (2018) have shown that they typically provide new information to users. Therefore, we assume that neutral and humanized how- 


\section{HUMANIZE, THEREFORE I UNDERSTAND?}

explanations increase the perceived and the objective user understanding of an intelligent algorithm:

- H1: Compared to the control condition, the neutral how-explanation leads to higher objective and perceived user understanding.

- H2: Compared to the control condition, the humanized explanation leads to higher objective and perceived user understanding.

In the context of intelligent algorithms, effectance motivation drives the individual's wish for effective system interaction and thus, reduces the feeling of uncertainty about an intelligent system. Waytz et al. (2010) have shown that humanization can satisfy effectance motivation, leading to a higher perceived understanding and predictability of a system. Therefore, we assume that humanization can increase perceived understanding but does not affect objective understanding as no new information about the intelligent algorithm is acquired in this process.

- H3a: Compared to the control condition, humanization leads to higher perceived user understanding but not a higher objective understanding.

Additionally, technical expertise, i.e., the available knowledge about algorithms, should moderate the degree of humanization as it becomes less likely that the user applies the knowledge about oneself or humans to an intelligent algorithm. Furthermore, based on the three factor-theory (Epley et al., 2007), we suggest that personality traits such as the need for cognition, desire for control, and chronic loneliness moderate the effect of humanization on the perceived user understanding of intelligent algorithms:

- H3b: This effect is moderated by technical expertise, need for cognition, desirability for control, and chronic loneliness.

We argue that explanations and humanization work as knowledge mechanisms leading to an equal amount of perceived understanding. Therefore, we derive the 
I HUMANIZE, THEREFORE I UNDERSTAND?

following set of hypotheses regarding the effects of both mechanisms on the perceived and objective user understanding.

- H4a: The neutral explanation, humanization, and the humanized explanation do not differ in their effect on perceived user understanding.

- $H 4 b$ : The neutral explanation and humanized explanation lead to higher objective understanding than humanization.

Previous studies indicate that explanations and humanization can increase trust. Accordingly, we assume the following hypothesis:

- H5: Compared to the control conditions, all other conditions lead to higher trust perception in the system.

Regarding transparency perceptions, previous studies indicate that explanations increase the perceived transparency of systems (Q. Li et al., 2020). Thus, we assume:

- H6: Compared to the control condition and humanization condition, the neutral explanation and humanized explanation lead to higher transparency perception.

Regarding other effects of humanization and both types of how-explanations on systems perceptions (e.g., satisfaction, perceived usefulness), we do not have further specific hypotheses. Thus, we investigate these relations through an explorative approach.

\section{Method}

The study was approved by the local ethical committee of the University of Duisburg-Essen. All participants gave informed consent. We pre-registered our study before the data collection on OSF: https://osf.io/mz528 
I HUMANIZE, THEREFORE I UNDERSTAND?

\section{Participants}

We recruited 450 participants from the U.K.-based crowd-sourcing platform Prolific.co. After removing participants due to missing data and manipulation checks, 416 participants were included in the final sample. Participants' age ranged from 18 to 74 years $(M=33.54, S D=11.94)$. Regarding gender, 260 participants identified as female, 152 as male, three participants had other gender identities, and one participant preferred not to give any information on gender. Most participants had at least a bachelor's degree as highest education $(n=155)$ or a high school degree $(n=148)$. The majority of participants use Google News at least once a week $(n=194)$ or daily $(n=$ 127; at least once a month $n=41$; at least once a year $n=12$; less than once a year $n=$ 7). Thirty-five participants have never used Google News before.

\section{Study design}

We conducted an online experiment employing a between-subject design using "group" as an independent variable with four conditions: (1) control (2) neutral howexplanation, (3) humanization, (4) humanized how-explanation.

In the control condition, participants were asked to describe their experience with Google News. In the neutral how-explanation condition, participants received a how-explanation on the inner mechanism of Google News. The explanation was based on public information on the inner working of Google News (e.g., from their official website, public patents). It was further complemented by a figure showing an overview of the ranking procedure. Afterward, participants were asked to explain the inner mechanisms of Google News in their own words. In the humanization condition, participants wrote a text about the news curation algorithms in which they actively humanized the system. The instruction was adapted from the task paradigm of Waytz 
I HUMANIZE, THEREFORE I UNDERSTAND?

and colleagues (2010).

Besides the explanation and humanization condition, we decided to add a humanized how-explanation condition to facilitate the humanization process to explore the effects of both knowledge mechanisms. In this humanized how-explanation condition, participants received a how-explanation that was enriched with human elements. In this explanation, the ranking algorithm was compared to an expert who wishes to choose the most relevant news for the user. An image of a human expert subsidized this explanation.

All explanations were pre-tested in terms of their comprehensibility. All explanations, instructions, and the dataset can be found in the supplementary material on OSF: https://osf.io/k34fx/

\section{Measures}

Our main dependent variables were the user understanding of intelligent systems (in this case, Google News). We measured perceived understanding using three selfcreated items ("I understand how the system works", "I understand how the news are delivered to me", "The order in which Google News presents news articles to me makes a lot of sense to me. "). Items were measured on a 5-point-Likert scale. The internal consistency of the items was good, Cronbach's $\alpha=0.83$.

To measure objective understanding, we created a quiz consisting of 17 true-orfalse statements about personal data usage and the inner working of Google News (Table 1). The statements were informed by the available public information about the inner working of Google News (e.g., FAQ page, news articles, public patent). To avoid ceiling and floor effects, we pre-tested the difficulty and comprehensibility of each statement. 
I HUMANIZE, THEREFORE I UNDERSTAND?

Table 1. Overview of statements for the measurement of objective understanding

\begin{tabular}{|c|c|c|}
\hline No. & Statement & True/False \\
\hline 1 & $\begin{array}{l}\text { Google News monitors my clicks on news articles and links in order to give me } \\
\text { news article recommendations }\end{array}$ & True \\
\hline 2 & $\begin{array}{l}\text { Google News only compares my personal online behaviour to that of other users } \\
\text { in order to make news recommendations. }\end{array}$ & False \\
\hline 3 & $\begin{array}{l}\text { Google News cannot know what news topics I am interested in if I do not } \\
\text { explicitly disclose this to the application. }\end{array}$ & False \\
\hline 4 & $\begin{array}{l}\text { Google News is fully automated, i.e., there are no humans who intentionally pick } \\
\text { out the news articles for me (except for the Newsstand function). }\end{array}$ & True \\
\hline 5 & $\begin{array}{l}\text { Google News uses tags, i.e., keywords for each news article, which are based on } \\
\text { the news article, my past online behaviour, and my interests, to determine the most } \\
\text { interesting news for me. }\end{array}$ & True \\
\hline 6 & $\begin{array}{l}\text { For each news article, Google News calculates a score to determine the } \\
\text { importance of the news article. The score is only based on my past behaviour and } \\
\text { my data. }\end{array}$ & False \\
\hline 7 & Google News can track my online behaviour continuously. & True \\
\hline 8 & $\begin{array}{l}\text { Google News cannot know the country that I am living in if I do not explicitly } \\
\text { disclose it to the application. }\end{array}$ & False \\
\hline 9 & $\begin{array}{l}\text { The grammar and spelling of a news article are factors that is considered by } \\
\text { Google News. }\end{array}$ & True \\
\hline 10 & $\begin{array}{l}\text { Google News does not consider my past online activities on other Google } \\
\text { platforms, such as YouTube or Google Search, to give me news article } \\
\text { recommendations. }\end{array}$ & False \\
\hline 11 & $\begin{array}{l}\text { Google News can have access to my voice recordings that were recorded when I } \\
\text { spoke to Google services (e.g., using the mic icon to search by voice). }\end{array}$ & True \\
\hline 12 & Google News uses my age to determine the most interesting news articles for me. & False \\
\hline 13 & $\begin{array}{l}\text { For Google News to show me more similar news articles that I will be interested } \\
\text { in, I can express this through the "Like" button in every news article I like. }\end{array}$ & True \\
\hline 14 & Google News uses the same ranking algorithms as Google Search. & True \\
\hline 15 & $\begin{array}{l}\text { If there is an advertisement in the news article, the news article is more likely to } \\
\text { be delivered to me. }\end{array}$ & False \\
\hline 16 & Google News considers my political beliefs to deliver news to me. & False \\
\hline 17 & $\begin{array}{l}\text { Google News uses my gender to determine the most interesting news articles for } \\
\text { me. }\end{array}$ & False \\
\hline
\end{tabular}




\section{HUMANIZE, THEREFORE I UNDERSTAND?}

In addition to the perceived and objective understanding, we measured the system perception of Google News. We were particularly interested in satisfaction, transparency perception, and perceived usefulness. For this, we used the respective subscales from ResQue (Pu et al., 2011). All items were measured on a 5-point-Likert scale $(1=$ strongly agree $; 5=$ strongly disagree $)$, including a "not applicable" option. The internal consistency of these subscales was acceptable to good, Cronbach's $\alpha=.68$ to .86 (Pu et al., 2011). Furthermore, we measured trust through ten items taken from Cramer et al. (2008), e.g., "I am confident in the system.". We measured these items on a 7-point-Likert scale. Internal consistency of this scale is reported to be excellent, Cronbach's $\alpha=.90$ (Cramer et al., 2008).

Besides these dependent variables, we further measured several moderators, which were discussed in the literature. We measured the technical expertise through two items which were taken from Lee and Baykal (2017). Here, technical expertise consisted of a rating of the programming knowledge and knowledge on computational algorithms. Both ratings were measured on a 4-point scale ("no knowledge", "a little knowledge", "some knowledge", "a lot of knowledge").

Finally, we measured several personality traits that are known to be related to humanization (Epley et al., 2007): The need for cognition was measured through a short version of the Need for Cognition Scale (NCS; Lins de Holanda Coelho et al., 2020). It consisted of six items (e.g., "I would prefer complex to simple problems). All items are measured on a 5-point Likert scale $(1=$ extremely uncharacteristic of me $; 5=$ extremely characteristic of me). The internal consistency of the measure is good and comparable to the original NCS-18 scale (Cacioppo et al., 2013), Cronbach's $\alpha=.86$ to .90 .

We measured the desirability of control using the scale by Burger and Cooper (1979). The scale consists of 20 items (e.g., "I prefer a job where I have a lot of control 
I HUMANIZE, THEREFORE I UNDERSTAND?

over what I do and when I do it."; "I enjoy political participation because I want to have as much of a say in running government as possible.") which are answered on a 7-pointLikert scale $(1=$ does not apply to me at all, $7=$ always applies to me $)$. The internal consistency is reported to be acceptable, Cronbach's $\alpha=.70$.

Finally, we measured chronic loneliness using the ULS-8 scale by Hays and DiMatteo (1987), which consists of eight items (e.g., "I lack companionship."). The items are measured on a 4-point Likert scale ( $1=$ I often feel this way; $4=$ I never feel this way). The internal consistency is good, Cronbach's $\alpha=.84$.

\section{Procedure}

The study was conducted in February 2021 on the survey platform Soscisurvey. First, we measured the demographics and moderators. Then, participants were instructed to think of Google News and their experience with it. Afterward, participants were randomly assigned to one of the four conditions. Depending on the condition, participants wrote their explanations on the inner working of the Google News, described a story about Google News, or were asked to write about their experience with the application. After this, we measured the perceived and objective understanding. Participants were debriefed and received 3.54 \$ as compensation for participation. They needed about 13 minutes on average to complete the online experiment.

\section{Results}

To answer our hypotheses regarding the perceived understanding, we originally pre-registered planned contrasts which considered all possible group comparisons. However, we conducted a one-way ANOVA as this procedure was more appropriate. Detailed reasoning for a changed strategy of analysis as well as the results of the originally pre-registered analysis can be found on OSF. Concerning the remaining 


\section{HUMANIZE, THEREFORE I UNDERSTAND?}

hypotheses, we strictly followed our pre-registered strategy of analysis and conducted planned contrasts regarding the objective understanding and trust and moderation analyses. In addition, we performed an explorative analysis to examine the effect of the groups on the system perceptions.

We used IBM SPSS 27 and PROCESS v. 3.5. to perform the statistical analyses. For all analyses, Levene's test indicated that homogeneity of variance could be assumed. We applied Bonferroni-correction to all post-hoc comparisons.

\section{Perceived and objective understanding}

To analyze the effects on perceived understanding, we performed a one-way ANOVA using group as a factor. The omnibus-test was significant (Table 2$), F(3,412)$ $=11.82, p<.001$, partial $\eta^{2}=0.79,95 \%$ CI $[.03, .13]$.

Table 2. Overview of descriptive statistics for perceived and objective understanding

\begin{tabular}{llcccc}
\hline Condition & \multirow{2}{*}{$n$} & \multicolumn{2}{c}{ Perceived understanding } & \multicolumn{2}{c}{ Objective understanding } \\
\cline { 3 - 6 } & & \multicolumn{1}{c}{$M(S D)$} & $95 \%$ CI & $M(S D)$ & $95 \%$ CI \\
\hline Control & 110 & $3.39(.75)$ & {$[3.25,3.53]$} & $11.15(1.64)$ & {$[10.83,11.45]$} \\
Humanization & 112 & $3.51(.80)$ & {$[3.36,3.66]$} & $10.58(1.81)$ & {$[10.24,10.92]$} \\
Neutral Explanation & 90 & $3.84(.71)$ & {$[3.69,3.98]$} & $11.01(1.92)$ & {$[10.61,11.41]$} \\
Humanized Explanation & 104 & $3.90(.70)$ & {$[3.77,4.04]$} & $11.19(1.86)$ & {$[10.83,11.56]$} \\
\hline
\end{tabular}

Post-hoc comparisons revealed four significant group differences: Compared to the control group, participants in the neutral how-explanation condition had a significantly higher perceived understanding (95\% CI of the difference between means $[-.73,-.17], p<.001)$. Similarly, participants of the humanized how-explanation condition also had a significantly higher perceived understanding than the control group (95\% CI of the difference between means [-.79, -.25], $p<.001)$.

Additionally, we found that compared to the humanization condition, the neutral how-explanation condition $(95 \% \mathrm{CI}$ of the difference between means $[-.60,-.05], p=$ 


\section{HUMANIZE, THEREFORE I UNDERSTAND?}

.013 ) and the humanized how-explanation, respectively, showed significantly higher perceived understanding $(95 \% \mathrm{CI}$ of the difference between means $[-.66,-.12], p<$ .001). Thus, both explanations increased the perceived understanding significantly. Humanization, in turn, did not affect the perceived understanding.

To examine the effects on objective understanding, we conducted four planned contrasts (Figure 1). We note that for planned contrasts, the adjustments of p-values are controversial. We decided for the conservative adjustment of the significance level to $p$ $=0.05 / 4=0.0125($ Frane, 2015) .

First, we compared the control group with each experimental group separately. The planned contrast comparing the control condition with the neutral how-explanation was not significant $(t(412)=-0.52, p=.602$, Cohen's $d=-.076,95 \%$ CI for effect size $[-.35, .20])$. Furthermore, the planned contrast for the comparison between control condition and humanized how-explanations was not significant either $(t(412)=0.19, p$ $=.850$, Cohen's $d=.027,95 \%$ CI for effect size $[-.24, .29])$.

When comparing the control condition and the humanization condition, we found a decrease of objective understanding, $(t(412)=-2.33, p=.020$, Cohen's $d=-$ $.327,95 \% \mathrm{CI}$ for effect size $[-.59,-.06])$. While this result was not significant (according to our adjusted p-value), we still consider this result relevant due to the small overlap of confidence intervals and medium effect size. We performed four moderation analyses using perceived understanding as an outcome variable and need for cognition, desire for control, technical expertise, chronic loneliness as respective predictors. These analyses revealed no significant interaction effects.

Additionally, we found that, compared to the humanization condition, the neutral and humanized explanation lead to a significant higher objective understanding $(t(412)=2.43, p=.016$, Cohens' $d=.559,95 \%$ CI for effect size $[.09,1.02])$. 
Figure 1. Group means for objective understanding. Error bars represent 95\% confidence intervals of means.

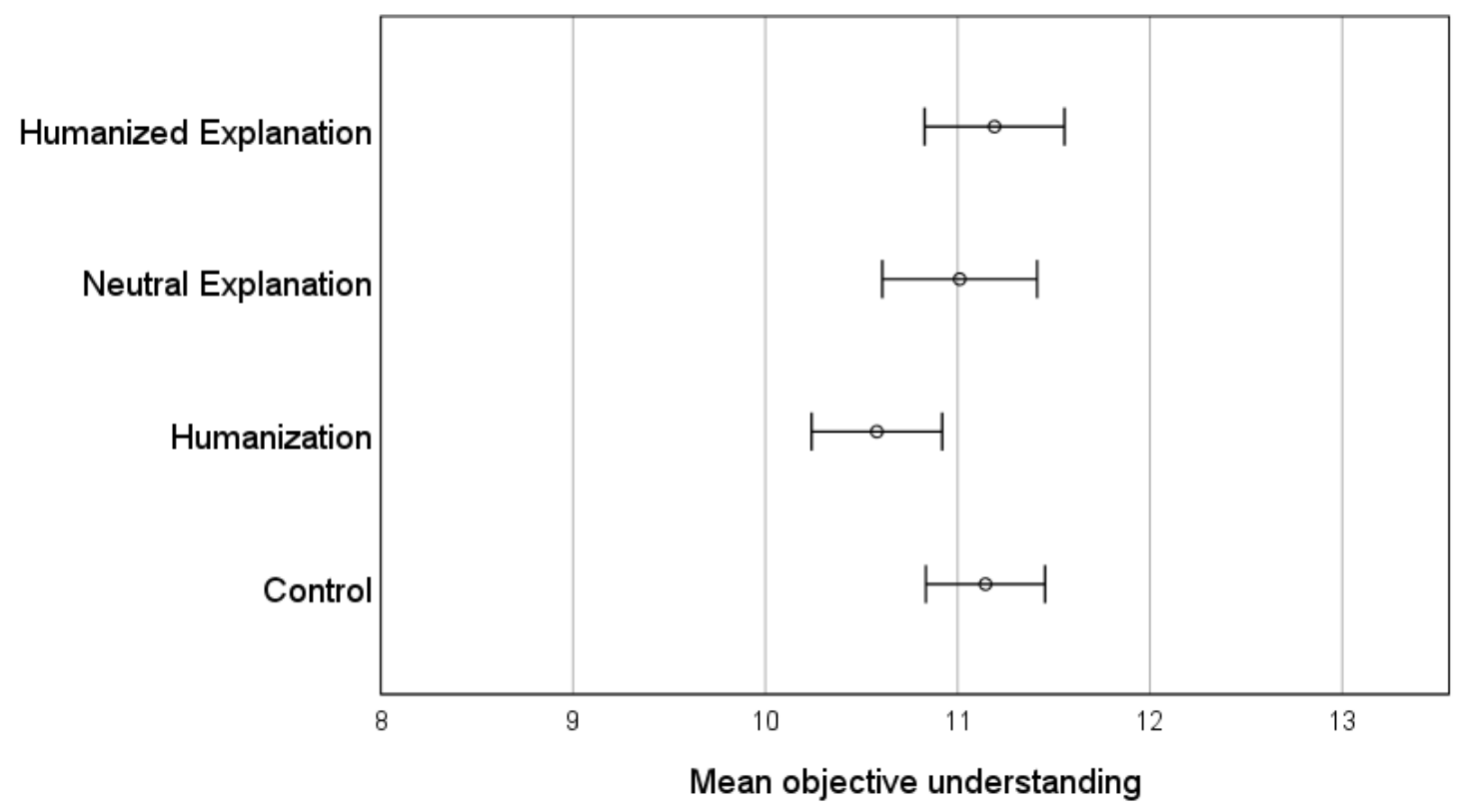

Note. Measurement of objective understanding consisting of 17 true-or-false statements. Thus, participants could achieve up to 17 correct answers.

In sum, we reject most of our hypotheses, H1-H4a. We found that both explanations lead to a higher perceived understanding. However, humanization did not lead to a higher perceived understanding. Our results supported H4b, suggesting that both explanations lead to higher objective understanding than humanization. However, participants who received (neutral or humanized) how-explanations did not perform significantly better than the control group. Surprisingly, humanization led to a relatively lower objective understanding than the control condition.

\section{System perceptions}

Regarding trust, the planned contrast revealed that compared to the neutral howexplanation, the humanized how-explanation and humanization did not increase trust 
perception significantly $(t(412)=0.47, p=.641$, Cohen's $d=.118,95 \%$ CI for effect size $[-.38, .61]$, Table 3$)$. Thus, we reject H5.

The last contrast (H6) revealed that compared to the control and humanization condition, both explanation conditions yielded in a significant increase of transparency perception $(t(403)=4.41, p<.001$, Cohen's $d=.878,95 \%$ CI for effect size $[0.48$, 1.27]). Therefore, results support H6. ${ }^{1}$

Table 3. Overview of descriptive statistics for system perceptions

\begin{tabular}{|c|c|c|c|c|}
\hline \multirow[t]{2}{*}{ Condition } & \multicolumn{2}{|c|}{ Trust } & \multicolumn{2}{|c|}{ Transparency } \\
\hline & $M(S D)$ & $95 \% \mathrm{CI}$ & $M(S D)$ & $95 \% \mathrm{CI}$ \\
\hline Control & $4.11(1.16)$ & {$[3.89,4.33]$} & $3.79(0.92)$ & {$[3.61,3.97]$} \\
\hline Humanization & $4.47(1.17)$ & {$[4.25,4.69]$} & $3.95(0.92)$ & {$[3.77,4.12]$} \\
\hline Neutral Explanation & $4.43(1.11)$ & {$[4.19,4.66]$} & $4.24(0.68)$ & {$[4.09,4.38]$} \\
\hline \multirow[t]{3}{*}{ Humanized Explanation } & $4.51(1.15)$ & {$[4.29,4.74]$} & $4.23(.078)$ & {$[4.08,4,38]$} \\
\hline & \multicolumn{2}{|c|}{ Perceived usefulness } & \multicolumn{2}{|c|}{ Satisfaction } \\
\hline & $M(S D)$ & $95 \% \mathrm{CI}$ & $M(S D)$ & $95 \% \mathrm{CI}$ \\
\hline Control & $3.56(.86)$ & {$[3.40,3.73]$} & $3.40(1.03)$ & {$[3.20,3.59]$} \\
\hline Humanization & $3.80(.84)$ & {$[3.65,3.96]$} & $3.63(1.09)$ & {$[3.42,3.84]$} \\
\hline Neutral Explanation & $3.81(.66)$ & {$[3.67,3.95]$} & $3.72(.95)$ & {$[3.52,3.92]$} \\
\hline Humanized Explanation & $3.92(.67)$ & {$[3.79,4.05]$} & $3.72(.93)$ & {$[3.54,3,90]$} \\
\hline
\end{tabular}

Moreover, we performed four one-way ANOVAs using group as an independent variable and trust, transparency, perceived usefulness, and satisfaction as respective measures. Results of the ANOVAs showed a significant omnibus-effect of group on trust, transparency, and perceived usefulness (Table 4).

\footnotetext{
${ }^{1}$ We note that regarding transparency, nine participants answered with "not applicable" leading to fewer data points for this measure (degree of freedom is therefore lower than for other measures).
} 
I HUMANIZE, THEREFORE I UNDERSTAND?

Table 4. One-way ANOVA and post-hoc comparisons for system perceptions

\begin{tabular}{lcccccc}
\hline & $F$ & $d f$ & $p$ & $\begin{array}{c}\text { partial } \\
\eta^{2}\end{array}$ & $\begin{array}{c}95 \% \\
\text { CI }\end{array}$ & $\begin{array}{c}\text { Post-Hoc } \\
\text { comparisons }\end{array}$ \\
\hline Trust & 2.78 & 3,412 & $.041^{*}$ & .020 & & \\
Transparency & 7.05 & 3,403 & $<$ & .050 & {$[-0.00$,} & $1<3,1<4$ \\
& & & $.001^{*}$ & & $0.09]$ & \\
Perceived & 4.04 & 3,407 & $.008^{*}$ & .029 & {$[0.00$,} & $1<4$ \\
usefulness & & & & & $0.06]$ & \\
Satisfaction & 2.38 & 3,405 & .069 & .017 & & \\
\hline Note: & & & & & &
\end{tabular}

Note: * significant at least at $p<.05 ; 1=$ control, $2=$ humanization, $3=$ neutral

explanation, 4 = humanized explanation.

Subsequent post-hoc comparisons revealed no significant group differences for trust but for transparency and perceived usefulness. Furthermore, we found that compared to the control condition, the neutral how-explanation (95\% CI of mean difference $[-.77,-.13], p=.001)$ and the humanized how explanation $(95 \%$ CI of mean difference $[-.75,-.13], p=.001)$ increased transparency perception significantly (Table $4)$.

Regarding perceived usefulness, we found a significant difference between the control group and the humanized how-explanation (95\% CI of mean difference [-.64, $.08], p=.005)$. Effect sizes of the one-way ANOVAs can be considered relatively large (Perugini et al., 2018).

In addition, we conducted further exploratory analyses of moderators and possible relationships to objective understanding and system perceptions. Here, in all moderation analyses, we used group as the predictor variable. For the moderation analyses, we used the need for cognition, desirability for control, technical expertise, and chronic loneliness as moderators and system perceptions (trust, transparency, 
satisfaction, perceived usefulness) as well as objective understanding as predictors.

This additional analysis revealed that transparency perception is moderated by technical expertise, $F(7,399)=4.33, p<.001, R^{2}=0.07$. In particular, we found a significant interaction effect between neutral explanation and control group, $b=.4558$, $t(399)=2.90, p=.004$. The addition of this interaction contributed to a significant change to the model, $F(3,399)=2.97, p=.0318, \Delta R^{2}=.0207$.

For participants scoring $1 \mathrm{SD}(-.66)$ below the mean of technical expertise, the difference between the neutral how-explanation and control group is .147 ( $p=.356)$. For participants scoring $1 \mathrm{SD}(.75)$ over the mean of technical expertise, the difference between the neutral how-explanation and the control group is .790 $(p<.001$; Figure 2).

Figure 2. Moderation of technical expertise on the relationship between groups and transparency perceptions

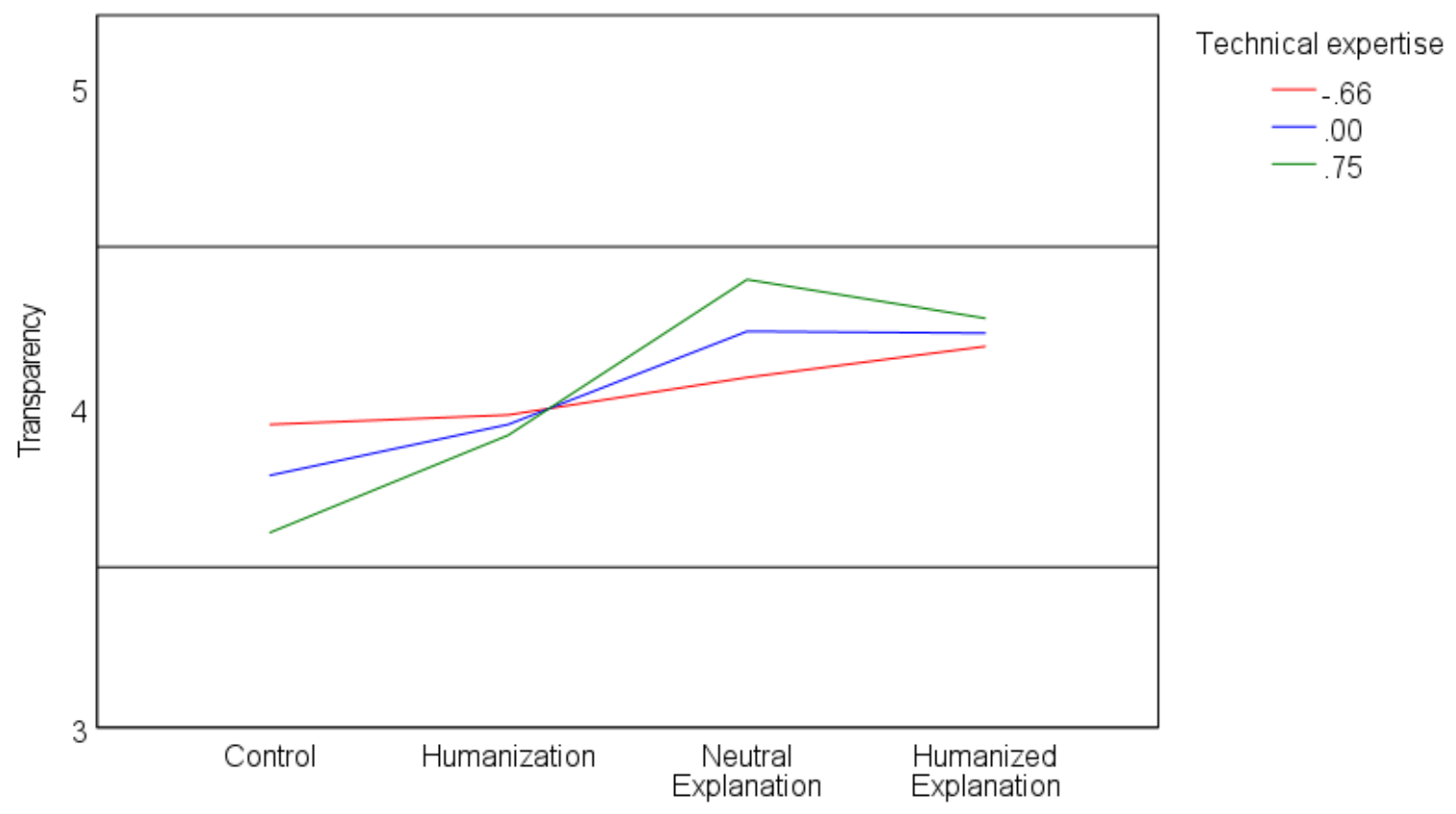


I HUMANIZE, THEREFORE I UNDERSTAND?

In sum, contrary to our expectation, humanization and the humanized howexplanation did not yield higher trust perception in the system. The further exploration of system perceptions revealed that both types of explanations led to higher transparency perceptions. We, additionally, found that this effect was moderated by technical expertise, in particular, in the neutral how-explanation condition. Finally, the humanized how-explanation led to higher perceived usefulness of Google News. None of the experimental conditions affected satisfaction with the system.

\section{Discussion}

We compared the effects of humanization, neutral and humanized howexplanations on perceived and objective user understanding, and system perceptions. Our main result is that, compared to the control and humanization conditions, both types of explanations resulted in a higher perceived understanding and transparency perception, but not in an increase of objective understanding. This result is particularly important as it shows that how-explanations can lead to a deceptive feeling of understanding: Users who receive how-explanations might view intelligent systems as transparent and might believe in having a certain user understanding of a system, while, in fact, they do not. Hence, we observed a discrepancy between the perceived understanding and objective understanding. One possibility is that the how-explanations used in this study might have failed their main purpose: transferring new knowledge to users (Keil, 2006). This has two implications for future research:

Firstly, we argue that future studies on explanations should not merely measure the perceived but also make an effort to measure the objective user understanding. We acknowledge that this is difficult as there are no universal benchmarks for it. In our view, the solution to this challenge is use-case-specific as intelligent algorithms vary in their application, input, and output data. 
I HUMANIZE, THEREFORE I UNDERSTAND?

Secondly, while both types of how-explanations presented in this study were detailed and understandable, but they were insufficient to transfer new knowledge to the participants. One possible reason could be that the explanations were not tailored to their understanding, i.e., too obvious and without new information about the intelligent system. We conclude that it is crucial to explore what users know about intelligent systems. This needs to be translated into user-informed explanations that consider the specific user understanding (Ngo \& Krämer, 2021).

\section{Effects of humanization on perceived and objective user understanding}

We made three interesting observations regarding humanization: (1) Our results show that the humanization condition led to a lower perceived understanding compared to both explanation conditions. (2) Surprisingly, humanization also led to a lower objective understanding than the control condition that did not receive any explanation. And (3) we found that the humanized how-explanations, i.e., the combination of both knowledge mechanisms, affected the measures differently than active humanization.

This first observation is contrary to what is predicted by the three-factor theory, which claims that humanization satisfies effectance motivation resulting in higher perceived understanding (Epley et al., 2007). The observation is also contrary to prior empirical findings by Waytz et al. (2010), which showed that individuals reported higher (perceived) understanding and predictability for humanized objects than nonhumanized ones.

One explanation might be the different amount of distinct knowledge representations concerning the objects that participants humanized. According to the three-factor theory, the humanization of non-human agents is less likely, when individuals have many distinct cognitive representations of these agents (Epley et al., 2007). In the former study by Waytz et al. (2010), participants were asked to humanize 


\section{HUMANIZE, THEREFORE I UNDERSTAND?}

objects, including technical devices (human-like robot, mobile alarm clock), geometrical shapes, and animals (dog). Some of these objects entail anthropomorphic cues (human-like robot, dog) encouraging humanization. For the others, individuals might have had less cognitive representations about the functioning, thus leading to more humanization than our study. In fact, for intelligent systems, prior research could show that users can hold distinct concept-based and processed-based components in their user understanding (Kunkel et al., in press; Ngo et al., 2020). These distinct and more mechanistic components might have decreased the likelihood of humanization.

Another explanation for this observation might be that the humanization of the intelligent system did not satisfy the participants' effectance motivation sufficiently. Compatible with this assumption, the humanization did not significantly affect the transparency perception. This indicates that, despite humanization, participants might have still felt uncertain about the intelligent system. However, we note that we did not measure the uncertainty or predictability of the intelligent system directly so that this issue remains open for future work.

Moreover, we found that humanization led to a lower objective understanding than the control group, who did not receive any explanation. We assume that humanization might have inhibited the retrieval of the participant's prior knowledge about the system, leading to more incorrect answers in the quiz compared to the control group. Against the background of the three-factor theory, this assumption might be plausible: Humanization is the process of ascribing one's own mental states to a nonhuman agent (Epley et al., 2007). Thus, if individuals are explicitly requested to humanize a system, they might favor the application of their own mental states instead of their objective understanding of the system. Thus, the retrieval of the (objective) user understanding is inhibited. 


\section{HUMANIZE, THEREFORE I UNDERSTAND?}

Finally, our third observation concerns the humanized how-explanation. We found that the humanized how-explanation (which encouraged humanization through its anthropomorphic description of the intelligent system) affected the perceived and objective user understanding in the same manner as the neutral how-explanations. This raises the question of why its effects differed from the effects of the humanization condition. One possible answer for this is that the humanization suggested through the humanized how-explanation was less relevant to the participants than the explanatory content about the functioning of the system. Participants were explicitly asked to report on the inner working of Google News. Thus, they might have focused on the processes of the systems instead of the humanization of the system. The effects of the humanize how-explanations might have been different if we had asked about a more general description of Google News. In this case, participants might have focused more on the anthropomorphic cues instead, possibly leading to a lower objective user understanding.

\section{Effects on systems perceptions}

In addition to the effects on perceived and objective user understanding, we examined the effects of humanization, and humanized and neutral how-explanations on trust, transparency, perceived usefulness, and satisfaction.

In line with some former studies, we found no relationship between trust and humanization and humanized how-explanation. Former studies that found this relationship investigated humanization slightly differently: They added anthropomorphic cues to the respective non-human agent (e.g., in the case of Waytz et al. (2014) name, gender, and voice to an autonomous vehicle). Here, in our study, we did not add any anthropomorphic cues and relied on the individual's tendency (in the humanization condition) or anthropomorphic cues to explain (humanized how- 


\section{HUMANIZE, THEREFORE I UNDERSTAND?}

explanation condition). It, therefore, appears to be crucial for the increase of trust that the non-human agent itself needs to own anthropomorphic cues.

Furthermore, we found that both how-explanations resulted in a higher transparency perception. Technical expertise moderated this effect: The neutral howexplanation resulted in a higher transparency perception, especially when their technical expertise was high. We conclude that explanations might be beneficial for users who already have high technical expertise. Interestingly, we did not find this effect for the humanized how-explanation. We assume that the humanized how-explanation was not perceived as technically accurate due to the anthropomorphic cues. Apparently, the humanized how-explanation highlighted rather practical aspects of the system increasing the perceived usefulness.

Finally, none of the experimental conditions led to a significant increase in satisfaction with intelligent systems, the mere undertaking of understanding is not sufficient for an increase. Here, we assume that interaction and experience with the system might contribute more to the satisfaction with an intelligent system.

\section{Limitations and future work}

Our study focuses on the explanation and humanization of one specific platform. Therefore, it remains open whether these results can be generalized for other intelligent systems. This particularly applies to the aspect of humanization: Users might be able to humanize other intelligent systems quite differently, leading to possibly more substantial inhibitory effects of humanization. Therefore, we suggest future work on other platforms and systems.

Additionally, we focused on one specific type of explanation, namely, howexplanations. These can also vary in their level of detail and form. It, therefore, remains 


\section{HUMANIZE, THEREFORE I UNDERSTAND?}

open to how different levels of detail or completely different types of explanations (e.g., why-explanations) can impact the perceived and objective user understanding.

Finally, as algorithmic transparency is highly relevant for the users' autonomy, the (increase of) objective user understanding is a crucial explanation goal. We suggest testing the effects of more user-informed explanations that consider the user understanding and therefore hold more explanatory power for users. These should aim to impact the objective user understanding successfully.

\section{Conclusion}

Our experiment revealed three main results regarding explanation and humanization: (1) Neutral and humanized how-explanations could lead to a deceptive feeling of understanding. In other words, individuals who receive how-explanations might perceive intelligent systems as transparent and think that they understand it, while, in fact, they do not. This undermines the main purpose of explanations, i.e., transferring new knowledge to users. (2) The effects of humanized how-explanations might be task-dependent. When individuals need to focus on the inner working of a system, they might rely less on anthropomorphic cues and more on the explanatory content of the explanation. Finally, (3) humanization can lower objective understanding compared to participants who did not receive any explanations. This has theoretical implications: Active humanization might be an inhibitory process that hinders the retrieval of knowledge about a system. In conclusion, this study points out the importance of objective user understanding as a benchmark for explanations and further highlights the need for explanations that consider the prior user understanding. 
I HUMANIZE, THEREFORE I UNDERSTAND?

\section{Acknowledgment}

This research was funded by the German Research Foundation (DFG) under grant No.

GRK 2167, Research Training Group 'User-Centred Social Media'.

\section{Disclosure statement}

No potential competing interest was reported by the authors 
I HUMANIZE, THEREFORE I UNDERSTAND?

\section{References}

Aggarwal, P., \& McGill, A. L. (2012). When Brands Seem Human, Do Humans Act Like Brands? Automatic Behavioral Priming Effects of Brand Anthropomorphism. Journal of Consumer Research, 39(2), 307-323. https://doi.org/10.1086/662614

Araujo, T., Helberger, N., Kruikemeier, S., \& de Vreese, C. H. (2020). In AI we trust? Perceptions about automated decision-making by artificial intelligence. $A I \&$ SOCIETY, 35(3), 611-623. https://doi.org/10.1007/s00146-019-00931-w

Blut, M., Wang, C., Wünderlich, N. V., \& Brock, C. (2021). Understanding anthropomorphism in service provision: A meta-analysis of physical robots, chatbots, and other AI. Journal of the Academy of Marketing Science. https://doi.org/10.1007/s11747-020-00762-y

Burger, J. M., \& Cooper, H. M. (1979). The desirability of control. Motivation and Emotion, 3(4), 381-393. https://doi.org/10.1007/BF00994052

Cacioppo, J. T., Petty, R. E., \& Kao, C. F. (2013). Need for cognition scale. Measurement Instrument Database for Social Science.

Cotter, K., \& Reisdorf, B. (2020). Algorithmic Knowledge Gaps: A New Dimension of (Digital) Inequality. International Journal of Communication, 14, 21.

Cramer, H., Evers, V., Ramlal, S., van Someren, M., Rutledge, L., Stash, N., Aroyo, L., \& Wielinga, B. (2008). The effects of transparency on trust in and acceptance of a content-based art recommender. User Modeling and User-Adapted Interaction, 18(5), 455-496. https://doi.org/10.1007/s11257-008-9051-3

Darlington, K. (2013). Aspects of Intelligent Systems Explanation. Universal Journal of Control and Automation, 1(2), 40-51.

https://doi.org/10.13189/ujca.2013.010204 


\section{HUMANIZE, THEREFORE I UNDERSTAND?}

de Visser, E. J., Monfort, S. S., McKendrick, R., Smith, M. A. B., McKnight, P. E., Krueger, F., \& Parasuraman, R. (2016). Almost human: Anthropomorphism increases trust resilience in cognitive agents. Journal of Experimental Psychology: Applied, 22(3), 331-349. https://doi.org/10.1037/xap0000092

DeVito, M. A., Hancock, J. T., French, M., Birnholtz, J., Antin, J., Karahalios, K., Tong, S., \& Shklovski, I. (2018). The algorithm and the user: How can HCI use lay understandings of algorithmic systems? Extended Abstracts of the $2018 \mathrm{CHI}$ Conference on Human Factors in Computing Systems. https://doi.org/10.1145/3170427.3186320

Dodge, J., Liao, Q. V., Zhang, Y., Bellamy, R. K. E., \& Dugan, C. (2019). Explaining models: An empirical study of how explanations impact fairness judgment. Proceedings of the 24th International Conference on Intelligent User Interfaces, 275-285. https://doi.org/10.1145/3301275.3302310

Dominguez, V., Messina, P., Donoso-Guzmán, I., \& Parra, D. (2019). The effect of explanations and algorithmic accuracy on visual recommender systems of artistic images. Proceedings of the 24th International Conference on Intelligent User Interfaces, 408-416. https://doi.org/10.1145/3301275.3302274

Došilović, F. K., Brčić, M., \& Hlupić, N. (2018). Explainable artificial intelligence: A survey. 41st International Convention on Information and Communication Technology, Electronics and Microelectronics (MIPRO), 0210-0215.

Edwards, L., \& Veale, M. (2017). Slave to the algorithm? Why a right to an explanation is probably not the remedy you are looking for. Duke Law\& Technology Review, 16(1), 18-84.

Eiband, M., Buschek, D., Kremer, A., \& Hussmann, H. (2019). The Impact of Placebic Explanations on Trust in Intelligent Systems. Extended Abstracts of the 2019 
I HUMANIZE, THEREFORE I UNDERSTAND?

CHI Conference on Human Factors in Computing Systems, 1-6.

https://doi.org/10.1145/3290607.3312787

Epley, N., Akalis, S., Waytz, A., \& Cacioppo, J. T. (2008). Creating Social Connection Through Inferential Reproduction: Loneliness and Perceived Agency in Gadgets, Gods, and Greyhounds. Psychological Science, 19(2), 114-120. https://doi.org/10.1111/j.1467-9280.2008.02056.x

Epley, N., Waytz, A., Akalis, S., \& Cacioppo, J. T. (2008). When We Need A Human: Motivational Determinants of Anthropomorphism. Social Cognition, 26(2), 143-155. https://doi.org/10.1521/soco.2008.26.2.143

Epley, N., Waytz, A., \& Cacioppo, J. T. (2007). On seeing human: A three-factor theory of anthropomorphism. Psychological Review, 114(4), 864-886. https://doi.org/10.1037/0033-295X.114.4.864

Eslami, M., Karahalios, K., Sandvig, C., Vaccaro, K., Rickman, A., Hamilton, K., \& Kirlik, A. (2016). First I 'like' it, then I hide it. Proceedings of the 2016 CHI Conference on Human Factors in Computing Systems, 2371-2382. https://doi.org/10.1145/2858036.2858494

Eyssel, F., Kuchenbrandt, D., \& Bobinger, S. (2011). Effects of anticipated humanrobot interaction and predictability of robot behavior on perceptions of anthropomorphism. Proceedings of the 6th International Conference on HumanRobot Interaction - HRI '11, 61. https://doi.org/10.1145/1957656.1957673

Fisher, M., Goddu, M. K., \& Keil, F. C. (2015). Searching for Explanations: How the Internet Inflates Estimates of Internal Knowledge. Journal of Experimental Psychology: General, 144(3), 15. https://doi.org/10.1037/xge0000070 


\section{HUMANIZE, THEREFORE I UNDERSTAND?}

Forster, Y., Naujoks, F., \& Neukum. (2017). Increasing anthropomorphism and trust in automated driving functions by adding speech output. 2017 IEEE Intelligent Vehicles Symposium (IV), 365-372. https://doi.org/10.1109/IVS.2017.7995746

Frane, A. V. (2015). Planned Hypothesis Tests Are Not Necessarily Exempt From Multiplicity Adjustment. Journal of Research Practice, 11(1), 17.

Friedrich, G., \& Zanker, M. (2011). A Taxonomy for Generating Explanations in Recommender Systems. AI Magazine, 32(3), 90. https://doi.org/10.1609/aimag.v32i3.2365

Gillespie, T. (2014). The Relevance of Algorithms. In T. Gillespie, P. J. Boczkowski, \& K. A. Foot (Eds.), Media Technologies (pp. 167-194). The MIT Press. https://doi.org/10.7551/mitpress/9780262525374.003.0009

Golossenko, A., Pillai, K. G., \& Aroean, L. (2020). Seeing brands as humans: Development and validation of a brand anthropomorphism scale. International Journal of Research in Marketing, 37(4), 737-755. https://doi.org/10.1016/j.ijresmar.2020.02.007

Goudey, A., \& Bonnin, G. (2016). Must smart objects look human? Study of the impact of anthropomorphism on the acceptance of companion robots. Recherche et Applications En Marketing (English Edition), 31(2), 2-20. https://doi.org/10.1177/2051570716643961

Gregor, S., \& Benbasat, I. (1999). Explanations from Intelligent Systems: Theoretical Foundations and Implications for Practice. MIS Quarterly, 23(4), 497. https://doi.org/10.2307/249487

Haas, C., \& Moussawi, S. (2020). Are Anthropomorphic Intelligent Agents More Intelligent? AMCIS 2020 Proceedings, 6. https://aisel.aisnet.org/amcis2020/ai_semantic_for_intelligent_info_systems/ai_s 
I HUMANIZE, THEREFORE I UNDERSTAND?

emantic_for_intelligent_info_systems/3/?utm_source=aisel.aisnet.org\%2Famcis 2020\%2Fai_semantic_for_intelligent_info_systems\%2Fai_semantic_for_intellig ent_info_systems\%2F3\&utm_medium=PDF\&utm_campaign=PDFCoverPages

Hancock, P. A., Billings, D. R., Schaefer, K. E., Chen, J. Y. C., de Visser, E. J., \& Parasuraman, R. (2011). A Meta-Analysis of Factors Affecting Trust in HumanRobot Interaction. Human Factors: The Journal of the Human Factors and Ergonomics Society, 53(5), 517-527. https://doi.org/10.1177/0018720811417254

Hautea, S., Munasinghe, A., \& Rader, E. (2020). 'That's Not Me': Surprising Algorithmic Inferences. Extended Abstracts of the 2020 CHI Conference on Human Factors in Computing Systems, 1-7. https://doi.org/10.1145/3334480.3382816

Hays, R., \& DiMatteo, M. R. (1987). A Short-Form Measure of Loneliness. Journal of Personality Assessment, 51(1), 69-81. https://doi.org/10.1207/s15327752jpa5101_6

Keil, F. C. (2006). Explanation and Understanding. Annual Review of Psychology, 57(1), 227-254. https://doi.org/10.1146/annurev.psych.57.102904.190100

Kodama, C., St. Jean, B., Subramaniam, M., \& Taylor, N. G. (2017). There's a creepy guy on the other end at Google!: Engaging middle school students in a drawing activity to elicit their mental models of Google. Information Retrieval Journal, 20(5), 403-432. https://doi.org/10.1007/s10791-017-9306-х

Kunkel, J., Ngo, T., Ziegler, J., \& Krämer, N. (in press). Identifying Group-Specific Mental Models of Recommender Systems: A Novel Quantitative Approach. IFIP Conference on Human-Computer Interaction. INTERACT 2021, Bari, Italy. 
I HUMANIZE, THEREFORE I UNDERSTAND?

Lee, M. K., \& Baykal, S. (2017). Algorithmic mediation in group decisions: Fairness perceptions of algorithmically mediated vs. Discussion-based social division. CSCW, 1035-1048. http://dx.doi.org/10.1145/2998181.2998230

Li, M., \& Suh, A. (2021). Machinelike or Humanlike? A Literature Review of Anthropomorphism in AI-Enabled Technology. Hawaii International Conference on System Sciences. https://doi.org/10.24251/HICSS.2021.493

Li, Q., Chu, S. L., Rao, N., \& Nourani, M. (2020). Understanding the Effects of Explanation Types and User Motivations on Recommender System Use. Proceedings of the Eighth AAAI Conference on Human Computation and Crowdsourcing, 8, 83-91. https://ojs.aaai.org/index.php/HCOMP/article/view/7466

Lim, B. Y., \& Dey, A. K. (2009). Assessing demand for intelligibility in context-aware applications. Proceedings of the 11th International Conference on Ubiquitous Computing, 10. https://doi.org/10.1145/1620545.1620576

Lim, B. Y., Dey, A. K., \& Avrahami, D. (2009). Why and Why Not Explanations Improve the Intelligibility of Context-Aware Intelligent Systems. Proceedings of the SIGCHI Conference on Human Factors in Computing Systems, 2119-2128. https://doi.org/10.1145/1518701.1519023

Lins de Holanda Coelho, G., Hanel, P. H., \& Wolf, L. J. (2020). The Very Efficient Assessment of Need for Cognition: Developing a Six-Item Version. Assessment, 27(8), 1870-1885. https://doi.org/10.1177/1073191118793208

Lipton, Z. C. (2017). The Mythos of Model Interpretability. ArXiv:1606.03490 [Cs, Stat]. http://arxiv.org/abs/1606.03490 
I HUMANIZE, THEREFORE I UNDERSTAND?

Mohseni, S., Zarei, N., \& Ragan, E. D. (2020). A Multidisciplinary Survey and Framework for Design and Evaluation of Explainable AI Systems. ArXiv:1811.11839 [Cs]. http://arxiv.org/abs/1811.11839

Natarajan, M., \& Gombolay, M. (2020). Effects of Anthropomorphism and Accountability on Trust in Human Robot Interaction. Proceedings of the 2020 ACM/IEEE International Conference on Human-Robot Interaction, 33-42. https://doi.org/10.1145/3319502.3374839

Ngo, T., \& Krämer, N. (2021). It's Just a Recipe?-Comparing Expert and Lay User Understanding of Algorithmic Systems. Technology, Mind, and Behavior, 2(4). https://doi.org/10.1037/tmb0000045

Ngo, T., Kunkel, J., \& Ziegler, J. (2020). Exploring Mental Models for Transparent and Controllable Recommender Systems: A Qualitative Study. Proceedings of the 28th ACM Conference on User Modeling, Adaptation and Personalization, 183191. https://doi.org/10.1145/3340631.3394841

Norman, D. A. (1983). Some Observations on Mental Models. In D. Gentner \& A. L. Stevens (Eds.), Mental Models (pp. 7-14). Psychology Press.

Nourani, M. (2019). The Effects of Meaningful and Meaningless Explanations on Trust and Perceived System Accuracy in Intelligent Systems. The Seventh AAAI Conference on Human Computation and Crowdsourcing, 7, 97-105. https://ojs.aaai.org/index.php/HCOMP/article/view/5284

Pan, B., Hembrooke, H., Joachims, T., Lorigo, L., Gay, G., \& Granka, L. (2007). In Google We Trust: Users' Decisions on Rank, Position, and Relevance. Journal of Computer-Mediated Communication, 12(3), 801-823. https://doi.org/10.1111/j.1083-6101.2007.00351.x 
I HUMANIZE, THEREFORE I UNDERSTAND?

Perugini, M., Gallucci, M., \& Costantini, G. (2018). A Practical Primer To Power Analysis for Simple Experimental Designs. International Review of Social Psychology, 31(1), 20. https://doi.org/10.5334/irsp.181

Phillips, E., Zhao, X., Ullman, D., \& Malle, B. F. (2018). What is Human-like?:

Decomposing Robots' Human-like Appearance Using the Anthropomorphic roBOT (ABOT) Database. Proceedings of the 2018 ACM/IEEE International Conference on Human-Robot Interaction, 105-113. https://doi.org/10.1145/3171221.3171268

Pu, P., Chen, L., \& Hu, R. (2011). A User-centric Evaluation Framework for Recommender Systems. Proceedings of the Fifth ACM Conference on Recommender Systems, 157-164. https://doi.org/10.1145/2043932.2043962

Rader, E., Cotter, K., \& Cho, J. (2018). Explanations as Mechanisms for Supporting Algorithmic Transparency. Proceedings of the 2018 CHI Conference on Human Factors in Computing Systems - CHI '18, 1-13. https://doi.org/10.1145/3173574.3173677

van Pinxteren, M. M. E., Wetzels, R. W. H., Rüger, J., Pluymaekers, M., \& Wetzels, M. (2019). Trust in humanoid robots: Implications for services marketing. Journal of Services Marketing, 33(4), 507-518. https://doi.org/10.1108/JSM-01-20180045

Wang, W., \& Benbasat, I. (2007). Recommendation Agents for Electronic Commerce: Effects of Explanation Facilities on Trusting Beliefs. Journal of Management Information Systems, 23(4), 217-246. https://doi.org/10.2753/MIS07421222230410

Waytz, A., Heafner, J., \& Epley, N. (2014). The mind in the machine: Anthropomorphism increases trust in an autonomous vehicle. Journal of 
I HUMANIZE, THEREFORE I UNDERSTAND?

Experimental Social Psychology, 52, 113-117.

https://doi.org/10.1016/j.jesp.2014.01.005

Waytz, A., Morewedge, C. K., Epley, N., Monteleone, G., Gao, J.-H., \& Cacioppo, J. T. (2010). Making sense by making sentient: Effectance motivation increases anthropomorphism. Journal of Personality and Social Psychology, 99(3), 410435. https://doi.org/10.1037/a0020240

Wu, E. Y., Pedersen, E., \& Salehi, N. (2019). Agent, Gatekeeper, Drug Dealer: How Content Creators Craft Algorithmic Personas. Proceedings of the ACM on Human-Computer Interaction, 3(CSCW), 1-27.

https://doi.org/10.1145/3359321

Yeomans, M., Shah, A., Mullainathan, S., \& Kleinberg, J. (2019). Making sense of recommendations. Journal of Behavioral Decision Making, January. https://doi.org/10.1002/bdm.2118

Zanker, M., \& Ninaus, D. (2010). Knowledgeable Explanations for Recommender Systems. 2010 IEEE/WIC/ACM International Conference on Web Intelligence and Intelligent Agent Technology, 657-660. https://doi.org/10.1109/WIIAT.2010.131 